\title{
Clinical Laboratory Profile of Blood Culture Proven Enteric Fever in Children
}

\author{
Shah SC ${ }^{1}$, Neupane PS ${ }^{2}$, Guragain A $^{3}$, Dhakal AK
}

\section{Abstract}

Introduction: Enteric fever has diverse clinical presentations and laboratory findings and blood culture is gold standard for diagnosis in the children. This study was done to analyse clinical profile and laboratory findings in culture proven enteric fever.

Material and Methods: The study was descriptive observational study carried out in the patient aged 14 years of age or less admitted with culture proven enteric fever admitted in a Paediatric ward of a tertiary medical centre in Lalitpur, Nepal from April 2009 to February 2018.

Results: Forty children with blood culture proven were enrolled in the study. All children had fever with the mean duration of 5.3 days (1-14). The most common associated symptoms were gastrointestinal which included anorexia (47.5\%), pain abdomen (37.5\%), vomiting (37.5\%), diarrhoea (15\%) and constipation (5\%). Splenomegaly (25\%) and hepatomegaly $(17.5 \%)$ were the commonest signs. The majority of children (80\%) had normal total leucocyte count and $32.5 \%$ of them had anaemia. There were no children with thrombocytopenia. Salmonella typhi and Salmonella paratyphi A were isolated in $70 \%$ and $30 \%$ of children respectively. None of the isolates showed drug resistance against ceftriaxone, chloramphenicol, cotrimoxazole, gentamicin, norfloxacin and ofloxacin. There was no mortality.

Conclusions: Enteric fever should be suspected in all the children with fever for more than five days along with anorexia, gastrointestinal associated symptoms and normal white blood counts.

Key words: Nepal; salmonella typhi; typhoid fever

\section{Introduction}

E nteric fever, also known as typhoid fever, is a multisystemic febrile illness caused by Salmonella enterica serotype Typhi and Salmonella enterica serotypes Paratyphi A, B, or C. The incidence is estimated to be more than 100 cases per 100,000 person years in Southeast Asia including Nepal. ${ }^{1}$ The higher rates of typhoid fever have been reported in school children aged more than five years. ${ }^{2}$ There are diverse clinical presentations of enteric fever in children,
${ }^{1}$ Dr. Subhash Chandra Shah, MBBS, MD, Lecturer; ${ }^{2}$ Dr. Prashank Shree Neupane, MBBS, Intern; ${ }^{3}$ Dr. Anusmriti Guragain, MBBS, Intern; ${ }^{4}$ Dr. Ajaya Kumar Dhakal, MBBS, MD, Associate Professor. All from the Department of Paediatrics, KIST Medical College, Imadol, Lalitpur, Nepal.

\author{
Address for correspondence \\ Dr. Subhash Chandra Shah, \\ Lecturer, \\ Department of Paediatrics, \\ KIST Medical College, \\ Imadol Lalitpur, Nepal. \\ E-mail: subashshah2012@gmail.com
}

\author{
Acknowledgements: None \\ Funding: Nil \\ Conflict of Interest: None \\ Permission from IRB: Not Submitted
}

\section{How to cite}

Shah SC, Neupane PS, Guragain A, Dhakal AK. Clinical Laboratory Profile of Blood Culture Proven Enteric Fever in Children. J Nepal Paediatr Soc 2018;38(3):153-6.

doi: http://dx.doi.org/10.3126/jnps.v38i3.27512

Submitted on: 2020-01-02

Accepted on: 2020-02-08

This work is licensed under a Creative Commons Attribution 3.0 License.

\section{(c) (i)}


and it is not easy to differentiate clinically it from other febrile illnesses. Blood culture is the gold standard for diagnosis; however, culture technique, which is done in resource limited settings, with administration of prior antibiotics, leads to low yield of growth. In addition, it is ordinarily cultured from five to $10 \mathrm{ml}$ of blood in $30-50$ $\mathrm{ml}$ of broth, which limits the probability of recovering organisms in children. ${ }^{3}$ The Widal test which is a commonly performed serological test is of limited clinical utility in endemic areas like Nepal because positive results may also represent previous infection as well as cross infection.

There is a paucity of studies in literature in regards to incidence of blood culture, diagnostic challenge as wells complications of enteric fever in the Nepalese children. This study therefore will address these issues to diagnosis of enteric fever in children. From our study, we aimed to determine the common clinical and laboratory parameters in culture positive enteric fever in children which would perhaps guide in diagnosing and treating children with enteric fever in the future.

\section{Material And Methods}

The study was descriptive cross sectional study which included all the in-patient cases aged under 14 years with culture proven enteric fever admitted in the Paediatrics department of a tertiary care teaching institute in Lalitpur, Nepal. The study period was from April 2009 to February 2018. Convenience sampling technique was used for data collection. Ethics approval was obtained from the Institutional Review Committee (IRC) of the institute.

The children admitted in the ward with proven enteric fever with culture positivity were enrolled in the study. Demographic data of the culture positive children was noted. There were 40 children with culture proven enteric fever during the study period. The clinical presentation, laboratory findings, blood culture sensitivity pattern and outcome were noted and data were entered on standard pro forma.

Brain heart infusion broth was used for blood culture. The media was incubated aerobically at $37^{\circ}$ C. Subculture was done on MacConkey's agar, and blood agar media daily for one week and was checked for evidence of growth after one, two, three and seven days. Only those bottles showing signs of positive growth for days one, two and three had been cultured on agar plates. All bottles had been sub- cultured before being discarded as negative on day seven. Data analysis was done using SPSS version 20.0. Data has been summarised using percentages and proportions.

\section{Results}

Total of 40 children of culture proven enteric fever were found admitted in the paediatric ward during the study period. The mean age of the child was 6.3 years (six months to 14 years); three cases were below one year of age. Out of total, 22 (55\%) were females and 18 $(45 \%)$ were male.

All children had fever prior to admission with the mean duration of 5.3 days (1-14 days). The gastrointestinal symptoms were the most common as shown in the table (Table 1). The most common sign was splenomegaly (25\%) followed by hepatomegaly (17.5), only $15 \%$ of children had coated tongue. The physical findings are as summarised in table 2. The common complications are summarised in table 3 . None of the children had gastrointestinal bleeding/perforation, disseminated intravascular coagulation or cholecystitis as complications.

Table 1: Symptoms of enteric fever

\begin{tabular}{lcc}
\hline Symptoms & $\begin{array}{c}\text { Number of } \\
\text { cases }(\mathbf{n}=\mathbf{4 0})\end{array}$ & $\%$ \\
\hline Fever & 40 & 100 \\
\hline Anorexia & 19 & 47.5 \\
\hline Pain abdomen & 15 & 37.5 \\
\hline Vomiting & 15 & 37.5 \\
\hline Headache & 11 & 27.5 \\
\hline Cough & 9 & 22.5 \\
\hline Diarrhea & 6 & 15 \\
\hline Burning micturition & 4 & 10 \\
\hline Constipation & 2 & 5 \\
\hline
\end{tabular}

Table 2: Examination findings of enteric fever

\begin{tabular}{lcc}
\hline Examination findings & $\begin{array}{c}\text { Number of } \\
\text { cases }(\mathbf{n = 4 0 )}\end{array}$ & $\%$ \\
\hline Splenomegaly & 10 & 25 \\
\hline Hepatomegaly & 7 & 17.5 \\
\hline Coated tongue & 6 & 15 \\
\hline Pallor & 4 & 10 \\
\hline Nonspecific skin rashes & 1 & 2.5 \\
\hline Distended abdomen & 1 & 2.5 \\
\hline
\end{tabular}

Table 3: Complications of enteric fever

\begin{tabular}{lcc}
\hline Complications & $\begin{array}{c}\text { Number of } \\
\text { cases }(\mathbf{n}=\mathbf{4 0})\end{array}$ & $\%$ \\
\hline Pyelonephritis & 5 & 12.5 \\
\hline Meningitis with seizure & 2 & 5 \\
\hline Hepatitis & 2 & 5 \\
\hline Pneumonia & 2 & 5 \\
\hline Shock & 2 & 5 \\
\hline
\end{tabular}


WBC counts was within normal limit (4000-11000/ $\mathrm{cmm}$ ) in $80 \%$ of the patients. Fifteen percent of children had leucocytosis and leucopenia was seen in only $5 \%$ of children. Twenty five percent of children had neutrophilia. Lymphocytosis and lymphopenia were seen in 20\% and $12.5 \%$ of children respectively. Anaemia based on age-specific haemoglobin level was seen in $32.5 \%$ of children. Five children $(12.5 \%)$ cases had raised liver enzymes and none had thrombocytopenia $(<150,000$ / $\mathrm{cmm}$ ) or pancytopenia.

Salmonella typhi was isolated in $70 \%$ of children while Salmonella paratyphi A was isolated in remaining $(30 \%)$. None of the isolates showed drug resistance against ceftriaxone, chloramphenicol, cotrimoxazole, gentamicin, norfloxacin and ofloxacin. The isolates were resistant to nalidixic acid 15 out of 18 times (83.3\% resistance) and ciprofloxacin two out of 20 times (10\%). Intermediate sensitivity and full resistance to ampicillin was seen in four out of $25(16 \%)$ and one out of $25(4 \%)$ of the isolates respectively. All children were successfully managed with standard treatment protocol and discharged without any mortality.

\section{Discussion}

This study demonstrates that the gastrointestinal symptoms were the most common mode of presentation while splenomegaly is most common finding on examination in the children with enteric fever. Data from this study did in fact show various expected results in case of signs and symptoms of the condition, however there was a great variation in the lab parameters.

Studies done among the children suffering from enteric fever in the endemic regions of Asia and Africa have deduced fever to be the most persistent feature of paediatric enteric fever in 97\%-100\% cases ${ }^{4}$ and even in our study, the common among all symptoms was fever prior to admission with the mean duration of 5.3 days which is considered the duration for febrile response shown to the organism after exposure. ${ }^{5}$ There were no prolonged, unusual fever cases as reported by other literatures. 6,7

Enteric fever is a systemic illness usually associated with gastrointestinal symptoms in both children $^{8}$ and adults and our study also found multisystemic presentation.

Coated tongue was seen in only $15 \%$ of the children which is much lower than reported in a study in Maharastra, India. ${ }^{9}$ Other examination findings like splenomegaly and hepatomegaly were present only in $25 \%$ and $17.5 \%$. Overall, there was the lack of clinical signs when considering the diagnosis of enteric fever in children.

The common complications were found to be pyelonephritis $(12.5 \%)$, meningitis with seizure $(5 \%)$, hepatitis $(5 \%)$, pneumonia $(5 \%)$ and shock (5\%). Nevertheless, even during a prolonged duration of illness, there was no mortality noted. The major cause of mortality being gastrointestinal bleeding and perforation that requires a more vigorous immune system with well-primed Peyer's patches that develops with the increasing age, hence severe complications are less seen in children than in adults. ${ }^{4}$

In our study, majority of children (80\%) had total leucocyte count within normal limits (4000-11000/ $\mathrm{cmm}$ ) while $15 \%$ of children had leukocytosis which is a result quite contrary to that of adults but frequently seen in children with enteric fever. ${ }^{10}$ Anaemia based on age-specific haemoglobin level was seen in $32.5 \%$ of patients, a noteworthy haematological finding which can be attributed to bone marrow suppression or peripheral blood dyscrasias, ${ }^{11}$ or generally higher percentage of anaemia. A total of five children (12.5\%) had raised liver enzymes suggesting hepatic involvement also noted by several authors ${ }^{12,13}$ There were no children with thrombocytopenia $(<150,000 / \mathrm{cmm})$ which has been stated to be seen more commonly in children from Africa than those in Asia or even pancytopaenia. ${ }^{3}$

Many recent studies have shown increasing trend of Salmonella Paratyphi A infections in the endemic regions however with not much variation in clinical presentation from Salmonella typhi infection. ${ }^{14,15}$ This has been attributed to immunisation with the $\mathrm{Vi}$ antigen vaccine that does not protect against S. paratyphi, according to numerous researches. ${ }^{16,17}$ In our study as well, Salmonella typhi was isolated in $70 \%$ of children while Salmonella paratyphi A was also isolated in remaining $30 \%$ of children. This study supports the finding that majority of the isolates are Salmonella typhi followed by Salmonella paratyphi A.,18

The isolates were fully sensitive to ceftriaxone, chloramphenicol, cotrimoxazole, gentamicin, norfloxacin and ofloxacin. The re-emergence of sensitivity to conventional drugs chloramphenicol and cotrimoxazole was also reported by other studies conducted in Kathmandu valley. ${ }^{18,19}$ Long-term discontinuation of these conventional drugs from the clinical settings can be a reason of their re-emergence of sensitivity. ${ }^{18}$ Resistance to nalidixic acid was seen in most cases and few children were also resistant to ciprofloxacin, which lead to the use of ceftriaxone in all cases of enteric fever. Extensive use of fluoroquinolones in our clinical setting explains the emerging drug resistance. 
There were a few limitations to our study, some of which hindered certain parts of the study. Firstly, in our country where over the counter drugs have been thriving like wildflowers without a proper monitoring of the pharmacies. Doing so, antibiotics exploitation has undoubtedly aided the false negative results of blood culture, hence depriving our study of clinically diagnosed children with common presenting signs and symptoms. Secondly, it is not possible to conduct more expensive investigations that are more specific of enteric fever in our part of the world due to the economic condition of most of the patients. And being a single centric study, the

\section{References}

1. Crump JA, Luby SP, Mintz ED. The global burden of typhoid fever. Bull World Health Organ. 2004;82(5):34653. PMID: 15298225

2. Feasey NA, Archer BN, Heyderman RS, Sooka A, Dennis B, Gordon MA, et al. Typhoid fever and invasive nontyphoid salmonellosis, Malawi and South Africa. Emerging infectious diseases. 2010;16(9):1448.. DOI: 10.3201/eid1609.100125

3. Baker S, Favorov M, Dougan G. Searching for the elusive typhoid diagnostic. BMC Infect Dis. 2010;10:45. DOI: 10.1186/1471-2334-10-45.

4. Azmatullah A, Qamar FN, Thaver D, Zaidi AK, Bhutta ZA. Systematic review of the global epidemiology, clinical and laboratory profile of enteric fever. J Glob Health. 2015;5(2):020407. DOI: 10.7189/ jogh.05.020407

5. Thiem VD, Lin FY, Canh DG, Son NH, Anh DD. The Vi conjugate typhoid vaccine is safe, elicits protective levels of $\operatorname{lgG}$ anti- $\mathrm{Vi}$, and is compatible with routine infant vaccines. Clin Vaccine Immunol. 2011;18(5):7305. DOI: 10.1128/CVI.00532-10.

6. Dheer G, Kundra S, Singh T. Clinical and laboratory profile of enteric fever in children in northern India. Trop Doct. 2012;42(3):154-6. DOI: 10.1258/td.2012.110442.

7. Jog $S$, Soman $R$, Singhal $T$, Rodrigues $C$, Mehta $A$ Enteric fever in Mumbai-clinical profile, sensitivity patterns and response to antimicrobials. J Assoc Physicians India. 2008;56:237-40. PMID: 18702385

8. Pandey KK, Srinivasan S, Mahadevan S, Nalini P, Rao RS. Typhoid fever below five years. Indian Pediatr. 1990;27(2):153-6. PMID: 2361759

9. Siddiqui SS, Koppa SK, V KA. Clinical profile of typhoid fever in children at a tertiary care hospital: a cross sectional study. Int $\mathrm{J}$ Contemp Pediatr. 2017;4(6):1951-4.

10. Kumar S, Rizvi M, Berry N. Rising prevalence of enteric fever due to multidrug-resistant Salmonella: an epidemiological study. J Med Microbiol. 2008;57(10):1247-50. DOI: 10.1099/ jmm.0.2008/001719-0. findings may not be corroborated to the entire Nepalese children. Also, exclusion of clinically diagnosed children has unquestionably affected the results of our research.

\section{Conclusion}

In settings where blood culture yields low, our study entails us to raise a suspicion of enteric fever in all the children with fever for more than five days along with gastrointestinal symptoms and signs. Splenomegaly may be an important clue to enteric fever. The total leukocyte count may remain within normal limits. Further larger, multi centric studies are warranted to substantiate our findings

11. James J, Dutta TK, Jayanthi S. Correlation of clinical and haematology profiles with bone marrow responses in typhoid fever. Am J Trop Med Hyg. 1997;57(3):313-6. DOI: 10.4269/ajtmh.1997.57.313

12. StuartBM, Pullen RL. Typhoid; clinical analysis of 360 cases. Arch Intern Med (Chic). 1946 Dec;78(6):62961. DOI: $10.1001 /$ archinte.1946.00220060002001

13. Ramachandran S, Godfery JJ, Perera MVF. Typhoid hepatitis. JAMA 1974;230:236 - 240.

14. Karkey A, Arjyal A, Anders KL, Boni MF, Dongol $S$, Koirala $S$, et al.The burden and characteristics of enteric fever at a healthcare facility in a densely populated area of Kathmandu. PLoS One. 2010;5(11):e13988. DOI: 10.1371/journal. pone.0013988.

15. Maskey AP, Day JN, Phung QT, Thwaites GE, Campbell JI. Salmonella enterica serovar Paratyphi A and S. enterica serovar Typhi cause indistinguishable clinical syndromes in Kathmandu, Nepal. Clin Infect Dis. 2006;42(9):1247-53. DOI: 10.1086/503033

16. Rodrigues C, Shenai S, Mehta A. Enteric fever in Mumbai, India: the good news and the bad news. Clin Infect Dis. 2003;36(4):535. DOI: 10.1086/367644

17. Ochiai RL, Acosta CJ, Danovaro-Holliday M, Baiqing D, Bhattacharya SK, Agtini MD, et al. A study of typhoid fever in five Asian countries: disease burden and implications for controls. Bulletin of the world health organisation. 2008;86:260-8. DOI: 10.2471/blt.06.039818

18. Chand HJ, Rijal KR, Neupane B, Sharma VK, Jha B. Re-emergence of susceptibility to conventional first line drugs in Salmonella isolates from enteric fever patients in Nepal. J Infect Dev Ctries. 2014;8(11):1483-7. DOI: 10.3855/jidc.4228.

19. Maskey AP, Basnyat B, Thwaites GE, Campbell JI, Farrar JJ, Zimmerman MD. Emerging trends in enteric fever in Nepal: 9124 cases confirmed by blood culture 1993-2003. Trans R Soc Trop Med Hyg. 2008 Jan 1;102(1):91-5. DOI: 10.1016/j. trstmh.2007.10.003. 